\title{
Double Higgs Production in the High Energy Limit
}

\author{
Joshua Davies* \\ Institut für Theoretische Teilchenphysik, Karlsruhe Institute of Technology (KIT) \\ 76128 Karlsruhe, Germany \\ E-mail: joshua.davies@kit.edu
}

\section{Go Mishima}

Institut für Theoretische Teilchenphysik, Karlsruhe Institute of Technology (KIT)

76128 Karlsruhe, Germany,

Institut für Kernphysik, Karlsruhe Institute of Technology (KIT)

76344 Eggenstein-Leopoldshafen, Germany

E-mail: go.mishima@kit.edu

\section{Matthias Steinhauser}

Institut für Theoretische Teilchenphysik, Karlsruhe Institute of Technology (KIT) 76128 Karlsruhe, Germany

E-mail: matthias.steinhauser@kit.edu

\section{David Wellmann}

Institut für Theoretische Teilchenphysik, Karlsruhe Institute of Technology (KIT) 76128 Karlsruhe, Germany

E-mail: david.wellmannekit.edu

In this talk we summarize our work on NLO QCD corrections to the amplitude for double-Higgs boson production in the gluon fusion channel. We describe our setup to reduce the Feynman integrals of the amplitude to a minimal set of master integrals, and how we compute them. Finally we remark on how so-called uniformly transcendental integrals can be identified in the context of expansion by regions, which we have used extensively in our computation of boundary conditions for systems of differential equations for the master integrals.

Loops and Legs in Quantum Field Theory (LL2018)

29 April 2018 - 04 May 2018

St. Goar, Germany

\footnotetext{
*Speaker.
} 


\section{Introduction}

Double-Higgs boson production will be an important process to study in the coming years. As the simplest process which depends on the triple-Higgs coupling, the experimental measurement of double-Higgs amplitudes will give crucial insight into the electro-weak sector of our models of particle physics. Despite its small cross section, it is anticipated that double-Higgs production in gluon fusion will be observable after the coming high-luminosity upgrade of the Large Hadron Collider at CERN.

The leading-order (one-loop) contributions to the $g g \rightarrow H H$ process were computed many years ago [1,2]. Since then, there have been various efforts to improve our theoretical understanding of this process by investigating two-loop (NLO) QCD contributions. Although a full, analytic calculation of the amplitude has not been completed at NLO, it has been computed in the large- $m_{t}$ limit using an effective theory in Ref [3] and then checked and extended in Refs. [4, 5], where an asymptotic expansion of the full theory was performed. Large- $m_{t}$ contributions are also available at three loops (NNLO) in Refs. [6, 7, 8, 9]. A numerical computation of the full amplitude, exact in $m_{t}$, is available in Refs. [10,11]. Recently, results also became available in the limit of small Higgs transverse momentum [12].

These proceedings summarize a talk presented at Loops and Legs in Quantum Field Theory 2018 and a previous publication on these works [13], in which we aim to supplement existing results by providing the so-far unknown high-energy limit of the $g g \rightarrow H H$ virtual amplitude. Assigning particle momenta as $g\left(q_{1}\right) g\left(q_{2}\right) \rightarrow H\left(q_{3}\right) H\left(q_{4}\right)$, this virtual amplitude can be written in terms of the Mandelstam variables

$$
s=\left(q_{1}+q_{2}\right)^{2}, \quad t=\left(q_{1}+q_{3}\right)^{2}, \quad u=\left(q_{2}+q_{3}\right)^{2},
$$

where all momenta are incoming. In the high-energy limit we set all $q_{i}^{2}=0$ (and so $m_{H}^{2}=0$ ), and have that $s+t+u=0$. The Mandelstam variables become

$$
s=2 q_{1} \cdot q_{2}, \quad t=2 q_{1} \cdot q_{3}, \quad u=2 q_{2} \cdot q_{3}=-s-t ;
$$

the amplitude and master integrals can be written in terms of the variables $s, t, m_{t}^{2}$. We decompose the amplitude in terms of two independent Lorentz structures $A_{i}^{\mu v}$, multiplied by two form factors $\mathscr{M}_{i}$

$$
\mathscr{M}=\varepsilon_{1, \mu} \varepsilon_{2, v} \mathscr{M}^{\mu v}=\varepsilon_{1, \mu} \varepsilon_{2, v}\left(A_{1}^{\mu v} \mathscr{M}_{1}+A_{2}^{\mu v} \mathscr{M}_{2}\right),
$$

where $\varepsilon_{1}$ and $\varepsilon_{2}$ are the polarization vectors of the gluons. $\mathscr{M}_{1}$ and $\mathscr{M}_{2}$ can be projected from $\mathscr{M}^{\mu v}$ by means of projection operators,

$$
\mathscr{M}_{i}=P_{i, \mu v} \mathscr{M}^{\mu v} .
$$

We do not typeset $A_{i}^{\mu v}$ or $P_{i, \mu \nu}$ explicitly here, instead referring the reader to Ref [13]. In the following sections, we describe our computation of the form factors $\mathscr{M}_{i}$.

\section{Reduction}

The two-loop virtual amplitude is generated using qgraf [14]. The programs q2e and $\exp [15,16]$ map each Feynman diagram onto an integral topology, and convert the notation for 
further processing with FORM 4.2 [17]. For each diagram, colour factors and Dirac traces are computed, and a projection onto the form factors $\mathscr{M}_{i}$ is performed as described in Section 1. The Feynman integrals are then written as linear combinations of scalar Feynman integrals which we reduce to a minimal set of master integrals by making use of FIRE 5 .2 [18]. LiteRed [19, 20] is also used to provide symmetry information to FIRE. No approximations (other than $m_{H}^{2}=0$ ) are made at this point; the exact dependence on $m_{t}$ is retained.

After reduction, the amplitude is written in terms of 10 one-loop and 221 two-loop master integrals, however this is not in fact a minimal two-loop set. In order to expose additional relations between these 221 integrals, we make use of the FIRE command FindRules. By applying it to the input set of integrals from the amplitude and then applying the reduction relations, we form a set of equations which should hold if the reduction relations are consistent,

$$
\text { FindRules }[\mathrm{I}]==\mathrm{I} \text {. }
$$

Any of these equations which are not satisfied provide relations between some of the 221 master integrals. Using these additional relations we obtain a list of 161 two-loop master integrals, which are listed in Ref [13].

\section{Differential Equations}

We use the method of differential equations [21, 22] to compute the master integrals. The differential equations are solved by using an ansatz, suitable to describe the integrals in the highenergy limit. By differentiating the vector of master integrals with respect to a parameter $x \in$ $\left\{s, t, m_{t}^{2}\right\}$ and applying reduction relations to the result, one obtains a closed system of coupled first order differential equations for the vector of master integrals MI,

$$
\frac{\mathrm{d}}{\mathrm{d} x} \mathbf{M I}=K_{x} \cdot \mathbf{M I}
$$

where $K_{x}$ is a square matrix whose entries depend on $s, t, m_{t}^{2}$ and $\varepsilon$. Our high-energy (large- $m_{t}$ limit) ansatz for the an $l$-loop master integral $I$ has the following form, guided by the structure of an asymptotic expansion,

$$
I=\sum_{n_{1}=n_{1}^{\min }}^{n_{1}^{\max }} \sum_{n_{2}=n_{2}^{\min }}^{n_{\max }^{\max }} \sum_{n_{3}=0}^{2 l+n_{1}} C_{I, n_{1}, n_{2}, n_{3}}(s, t) \varepsilon^{n_{1}}\left(m_{t}^{2}\right)^{n_{2}}\left(\log m_{t}^{2}\right)^{n_{3}} .
$$

When combined with suitable boundary conditions, the differential equations can be integrated to determine the free coefficients of the ansatz, the functions $C_{I, n_{1}, n_{2}, n_{3}}$. The leading term in the expansion provides these boundary conditions; that is, the term with $n_{2}=n_{2}^{\min }$. This leading term is computed by using the method of expansion by regions [23, 24]. Each integral can be written as the sum of integrals in a hard-scaling region and several soft-scaling regions. These integrals are written as Mellin-Barnes integrals whose integrands either are independent of, or depend on, $t(s$ can be set to, say, -1$)$.

The $t$-independent case is easier to deal with; we evaluate the Mellin-Barnes integral numerically, to high precision (300 digits), and fit the result to a basis of irrational numbers using the PSLQ algorithm [25]. A further 200 digits are used to check the result. For the $t$-dependent case we can proceed in two ways: 
1. Set $t$ to a value, say, -1 . Evaluate the integral using PSLQ as in the $t$-independent case. The $t$ dependence of the boundary condition can be recovered by substituting it into the $t$ differential equation; this produces a coupled system of differential equations for the ansatz coefficients $C_{I, n_{1}, n_{2}^{\min }, n_{3}}(-1,-1)$, which can be integrated to obtain $C_{I, n_{1}, n_{2}^{\min }, n_{3}}(t,-1)$.

2. Construct a Taylor series for the Mellin-Barnes integral about $t=0$, by taking its residues. Fit the series to a basis of harmonic polylogarithms [26] to produce a $t$-dependent boundary condition; that is, the coefficients $C_{I, n_{1}, n_{2}^{\min }, n_{3}}(t,-1)$ are produced directly.

In both cases, we obtain $t$-dependent boundary conditions which can be substituted into the $m_{t}^{2}$ differential equations. Since all $m_{t}^{2}$ dependence is explicit in the ansatz, one obtains a system of linear equations relating the set of coefficients $C_{I, n_{1}, n_{2}, n_{3}}(t,-1)$ which can be solved, in principle, to an arbitrarily high maximum value of $n_{2}=n_{2}^{\max }$. In practice, higher values of $n_{2}^{\max }$ are computationally more difficult. We compute a sufficient number of terms to verify the convergence of the series. For more details on how the above steps are performed, the reader is referred to Ref [13].

After reconstructing the $s$ dependence on dimensional grounds, we obtain asymptotic expansions for the master integrals for small values of $m_{t}^{2}$. These expansions can be substituted into the amplitude. The leading two orders of these expansions are also given in Ref. [27], and we find complete agreement.

\section{Higgs Mass Effects}

After computing the master integrals using the method described in Section 3, we consider Higgs mass corrections to the amplitude in the high energy limit. Unreduced integrals appearing initially in the amplitude can be expanded as a Taylor series in $m_{H}^{2}$, since the Higgs bosons are external particles and their momenta can always flow along massive lines. This expansion can be performed with LiteRed. The resulting expression contains extra integrals which can be reduced, now for vanishing $m_{H}^{2}$, as described in Section 2. For example, we have that

$$
\begin{aligned}
\hat{F}_{6}(1,1,1,1,1,1,0,0) \rightarrow G_{6} & (1,1,1,1,1,1,0,0)+\frac{m_{H}^{2}}{s+t}\left\{G_{6}(1,1,1,1,1,1,1,0,0)\right. \\
& +\left(\frac{s}{2}-m_{t}^{2}\left(1+\frac{s}{2 t}\right)+t\right) F_{6}(1,1,2,1,1,1,1,0,0) \\
& -\left(1+\frac{s}{2 t}\right) F_{6}(1,1,2,1,1,1,1,0,-1)-\frac{s}{2 t} F_{6}(1,1,1,1,1,2,1,-1,0) \\
& -\left(\frac{s}{2}+m_{t}^{2} \frac{s}{2 t}\right) G_{6}(1,1,1,1,1,2,1,0,0)-\frac{1}{2} F_{6}(1,1,2,0,1,1,1,0,0) \\
& -\frac{1}{2} F_{6}(1,1,1,1,0,2,1,0,0)-\frac{1}{2} F_{6}(0,1,1,1,1,2,1,0,0) \\
& \left.+\left(\frac{3}{2}+\frac{s}{t}\right) F_{6}(1,0,2,1,1,1,1,0,0)\right\}+\mathscr{O}\left(\left(m_{H}^{2}\right)^{2}\right) .
\end{aligned}
$$

Integrals labelled with $F$ rather than $G$ are not master integrals, and will be reduced to our master integral basis. Hatted integrals denote that they are $m_{H}^{2}$ dependent. The definitions of our integral families can be found in Ref. [13]. 
Figure 1 shows the effect of including terms proportional to $m_{H}^{2}$ and $\left(m_{H}^{2}\right)^{2}$ in the differential cross-section at one loop. In the high-energy region where the $m_{t}^{2}$ expansion converges $(\sqrt{s} \gtrsim$ $800 \mathrm{GeV}$ ), the correction due to the $m_{H}^{2}$ terms describes the known exact result with an error below $1.5 \%$ (and an error below $0.1 \%$ for $\sqrt{s} \gtrsim 1000 \mathrm{GeV}$ ). In this region, the correction due to the $\left(m_{H}^{2}\right)^{2}$ terms is very small; it shifts the $m_{H}^{2}$ curve by less than $0.05 \%$. We thus have confidence that our asymptotic expansions describe the amplitude very well in the high-energy region, and feel that computing only the $m_{H}^{2}$ correction will be sufficient at NLO.

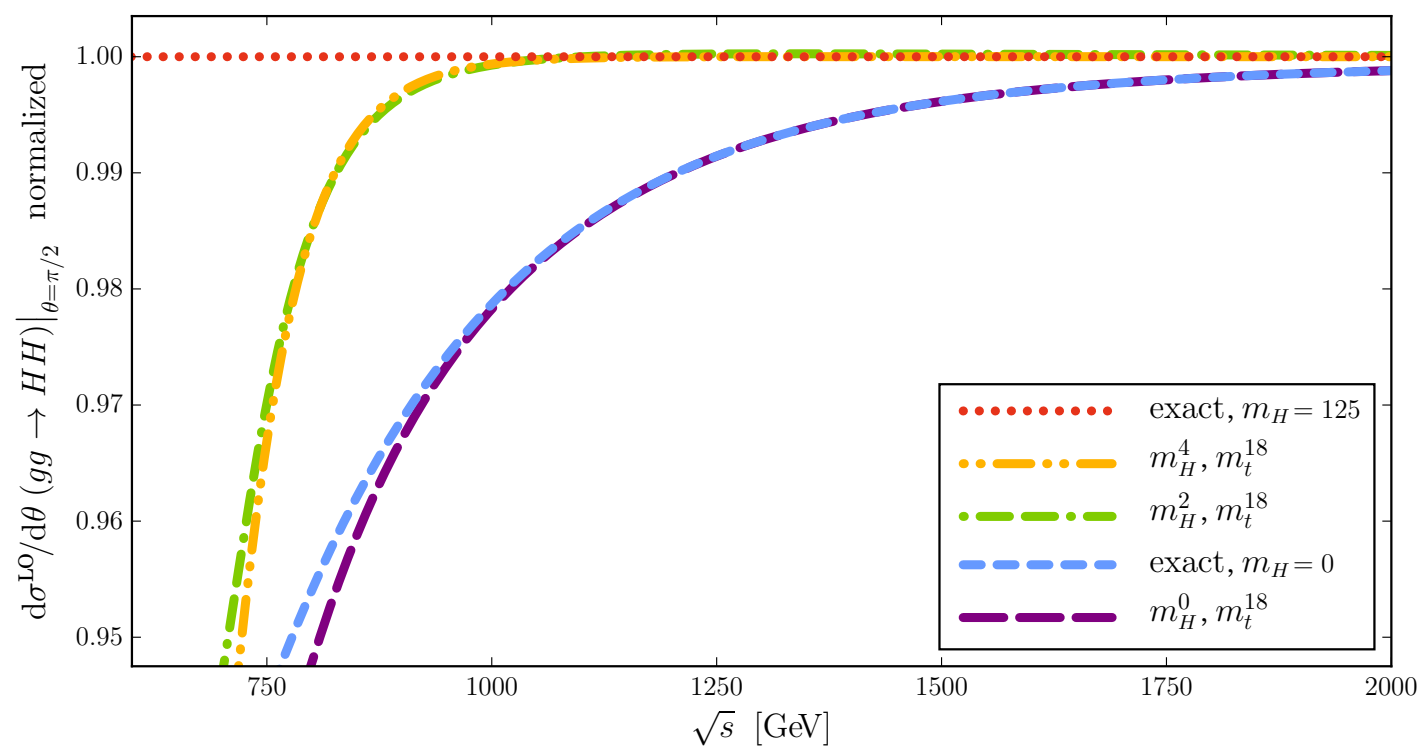

Figure 1: Various high-energy approximations of the LO differential cross section, normalized to the known, exact result. The curves are plotted for a Higgs-boson scattering angle $\theta=\pi / 2$. The curves labelled "exact"

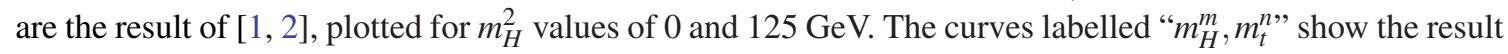
of our asymptotic expansion, including terms to $m, n$-th order in $m_{H}, m_{t}$.

\section{Expansion by Regions and Uniform Transcendentality}

Although we have not made use of the property of uniform transcendentality in our choice of basis for the planar master integrals, or for their calculation, we will describe some observations on how uniformly transcendental integrals can be identified by the method of expansion by regions. We proceed by example.

Consider the integral $G_{6}(1,1,1,1,1,1,1,0,0)$. It is a finite integral; that is, its expansion about $D=4-2 \varepsilon$ dimensions contains no poles in $\varepsilon$. Expanding by regions, where here the expansion parameter is $m_{t}$, one sees that the integral can be expressed as the sum of contributions from a hard region and several soft-collinear regions. The contribution from the hard region is simply the fully massless 2-loop double box integral, which has poles in $\varepsilon$ starting from $\varepsilon^{-4}$. These poles are cancelled by poles from the soft regions, such that we obtain a finite result.

This cancellation means that if the hard region is uniformly transcendental, as is the case for $G_{6}(1,1,1,1,1,1,1,0,0)$, the sum of the contributions from the soft regions must also be uniformly transcendental up to $\mathscr{O}\left(\varepsilon^{-1}\right)$. Indeed, the sum of the soft-collinear regions is also uniformly 
transcendental in its $\varepsilon^{0}$ and $\varepsilon^{1}$ terms, so we conclude that $G_{6}(1,1,1,1,1,1,1,0,0)$ is a uniformly transcendental integral, at least to the order in $\varepsilon$ required by our amplitude.

We emphasize that the uniform transcendentality of the first few terms of the $\varepsilon$-expansion of an integral does not guarantee that higher order terms of the series will be uniformly transcendental also. In such cases, one can seek linear combinations of integrals which are uniformly transcendental to all $\varepsilon$ orders required by the amplitude. For the 7-line planar master integrals considered here we have found the following uniformly transcendental basis,

$$
\begin{aligned}
& G_{6}(1,1,1,1,1,1,1,0,0), \\
& G_{6}(1,1,1,1,1,1,1,-1,0), \\
& G_{6}(1,1,1,1,1,1,1,-1,-1)+G_{6}(1,1,0,1,1,0,1,0,0), \\
& G_{20}(1,1,1,1,1,1,1,0,0), \\
& G_{20}(1,1,1,1,1,1,1,-1,0), \\
& G_{20}(1,1,1,1,1,1,1,0,-1), \\
& G_{20}(1,1,1,1,1,1,1,-1,-1)+G_{20}(1,1,0,1,1,0,1,0,0) .
\end{aligned}
$$

These combinations of integrals have been identified by first examining only the hard-scaling massless contributions, which is straightforward. Once a uniformly transcendental hard-scaling combination has been found, one can compute the more difficult soft contributions to check their transcendentality at the $\varepsilon$ powers required by the amplitude.

\section{Conclusions}

These proceedings have summarized our computation of the amplitude and planar master integrals of the $g g \rightarrow H H$ at NLO in QCD. We have computed these quantities in the high-energy limit, which is not well described by results currently available in the literature. We have found that in this limit, while corrections due to a non-zero $m_{H}^{2}$ are small, corrections due to $m_{t}^{2}$ are of crucial importance. Our work on the remaining non-planar master integrals is nearing completion, and the reader can expect a study on the size of these effects on the amplitude in the near future.

\section{References}

[1] E. W. N. Glover and J. J. van der Bij, Nucl. Phys. B 309 (1988) 282.

[2] T. Plehn, M. Spira and P. M. Zerwas, Nucl. Phys. B 479 (1996) 46 Erratum: [Nucl. Phys. B 531 (1998) 655] [hep-ph/9603205].

[3] S. Dawson, S. Dittmaier and M. Spira, Phys. Rev. D 58 (1998) 115012 [hep-ph/9805244].

[4] J. Grigo, J. Hoff, K. Melnikov and M. Steinhauser, Nucl. Phys. B 875 (2013) 1 [arXiv:1305.7340 [hep-ph]].

[5] G. Degrassi, P. P. Giardino and R. Gröber, Eur. Phys. J. C 76 (2016) no.7, 411 [arXiv:1603.00385 [hep-ph]].

[6] D. de Florian and J. Mazzitelli, Phys. Lett. B 724 (2013) 306 [arXiv:1305.5206 [hep-ph]]. 
[7] D. de Florian and J. Mazzitelli, Phys. Rev. Lett. 111 (2013) 201801 [arXiv:1309.6594 [hep-ph]].

[8] J. Grigo, K. Melnikov and M. Steinhauser, Nucl. Phys. B 888 (2014) 17 [arXiv:1408.2422 [hep-ph]].

[9] J. Grigo, J. Hoff and M. Steinhauser, Nucl. Phys. B 900 (2015) 412 [arXiv:1508.00909 [hep-ph]].

[10] S. Borowka, N. Greiner, G. Heinrich, S. P. Jones, M. Kerner, J. Schlenk and T. Zirke, JHEP 1610 (2016) 107 [arXiv:1608.04798 [hep-ph]].

[11] S. Borowka, N. Greiner, G. Heinrich, S. P. Jones, M. Kerner, J. Schlenk, U. Schubert and T. Zirke, Phys. Rev. Lett. 117 (2016) no.1, 012001 Erratum: [Phys. Rev. Lett. 117 (2016) no.7, 079901] [arXiv:1604.06447 [hep-ph]].

[12] R. Bonciani, G. Degrassi, P. P. Giardino and R. Gröber, [arXiv:1806.11564 [hep-ph]].

[13] J. Davies, G. Mishima, M. Steinhauser and D. Wellmann, JHEP 1803 (2018) 048 [arXiv:1801.09696 [hep-ph]].

[14] P. Nogueira, J. Comput. Phys. 105 (1993) 279.

[15] R. Harlander, T. Seidensticker and M. Steinhauser, Phys. Lett. B 426 (1998) 125 [hep-ph/9712228].

[16] T. Seidensticker, [hep-ph/9905298].

[17] B. Ruijl, T. Ueda and J. Vermaseren, [arXiv:1707.06453 [hep-ph]].

[18] A. V. Smirnov, Comput. Phys. Commun. 189 (2015) 182 [arXiv:1408.2372 [hep-ph]].

[19] R. N. Lee, [arXiv:1212.2685 [hep-ph]].

[20] R. N. Lee, J. Phys. Conf. Ser. 523 (2014) 012059 [arXiv:1310.1145 [hep-ph]].

[21] A. V. Kotikov, Phys. Lett. B 254 (1991) 158.

[22] T. Gehrmann and E. Remiddi, Nucl. Phys. B 580 (2000) 485 [hep-ph/9912329].

[23] M. Beneke and V. A. Smirnov, Nucl. Phys. B 522 (1998) 321 [hep-ph/9711391].

[24] V. A. Smirnov, Springer Tracts Mod. Phys. 250 (2012) 1.

[25] H.R.P. Ferguson and D.H. Bailey, RNR Technical Report, RNR-91-032; H.R.P. Ferguson, D.H. Bailey and S. Arno, NASA Technical Report, NAS-96-005.

[26] E. Remiddi and J. A. M. Vermaseren, Int. J. Mod. Phys. A 15 (2000) 725 [hep-ph/9905237].

[27] K. Kudashkin, K. Melnikov and C. Wever, [arXiv:1712.06549 [hep-ph]]. 